\title{
Dringlichkeitspauschale: ein Schritt in die richtige Richtung
}

Schon bald nach der Einführung von TARMED zeigten sich gravierende Probleme bei der Anwendung der Positionen der Notfallpauschalen A-C, die - vor allem wegen des erlaubten Zeitrahmens - sehr eng definiert sind. Die paritätische Interpretationskommission (PIK) musste mehrmals Stellung nehmen und Einzelheiten klären. Anfang Mai 2005 beschloss die paritätische Tarifkommission (PTK), eine Arbeitsgruppe (AG) zur Erarbeitung einer Dringlichkeitspauschale zu gründen.

Basierend auf einem Vorschlag der Schweizerischen Gesellschaft für Allgemeinmedizin (SGAM) wurde die jetzige Lösung erarbeitet (siehe Information des Tarifdienstes auf Seite 96 dieser Ausgabe) und im August 2005 der Antrag der AG der PTK vorgelegt. Die Partner (santésuisse, Medizinaltarifkommission [MTK], H+ und FMH) mussten in den folgenden Monaten ständig neue Varianten der Taxpunktbewertung sowie deren Kostenfolge berechnen, bis die PTK am 10. November 2005 den heutigen Antrag akzeptierte und an das Leitungsgremium TARMED Suisse weiterleitete. Entschlüsse der PTK und des Leitungsgremiums (LG) müssen einstimmig gefällt werden.

Anlässlich der ersten Traktandierung an der Sitzung des LG am 23. November 2005 stellte nun santésuisse plötzlich ein Junktim her zwischen der Dringlichkeitspauschale und den Resultaten einer anderen AG; derjenigen nämlich, die Probleme bei der Verrechnung von diversem Material mittels Materialpauschalen berechnen sollte. Erst am 11. April 2006 (!) akzeptierte das Leitungsgremium den Antrag, der dann mit der Tarifversion 1.04. Anfang Juli dem Bundesrat zur Genehmigung vorgelegt wurde. Diese erfolgte am 22. November 2006 und TARMED suisse wurde am 5. Dezember 2006 informiert.

Die FMH-Delegation, die an den Verhandlungen teilnimmt, ist sich bewusst, dass die Dringlichkeitspauschale nicht alle Erwartungen erfüllt. Diese honoriert aber alle Ärztinnen und Ärzte, die bereit sind, in dringenden Fällen ausserhalb der regulären Sprechstunde - also innerhalb von 2 Stunden - Patienten zu behandeln. Sie ist Ausdruck der Anerkennung einer ärztlichen «Sonderleistung», die zu grossen Kosteneinsparungen im Notfallbereich führt. Durch diese können viele unnötige Konsultationen auf Notfallstationen der Spitäler, deren Abklärungen deutlich teurer sind, vermieden werden. Sie ist aber auch ein Mittel, den Notfalldienst wieder attraktiver zu machen.

Die grössten Nutzniesser werden die Grundversorger sein, jedoch auch die Spezialisten können die Position bei Erbringen der Leistung in Rechnung stellen. Einzig im Spitalbereich ist sie nicht anwendbar. Die Kostenträger haben die Öffnung der Position für die im Spital erbrachten Leistungen der Belegärzte strikte abgelehnt. Nicht zur Diskussion stand die sogenannte «Expresskonsultation» während der Sprechstundenzeit, die zu Missbrauch mit Volumenerhöhung und nachfolgender TPW-Absenkung geführt hätte.

Der Preis, der für die neue Position bezahlt werden musste, ist eine Absenkung der Taxpunktwerte der Notfallpauschalen $\mathrm{A}-\mathrm{C}$, da die Umsetzung kostenneutral erfolgen musste.

Trotz allem ist dies ein Schritt in die richtige Richtung. Anpassungen und Verbesserungen in der Zukunft sind möglich und auch nötig. Der Bundesrat will Ende 2007 über die Auswirkungen der neu eingeführten Dringlichkeitspauschale bezüglich Zielerreichung und Kostenentwicklung informiert werden. Er verlangt allenfalls auch Änderungen.

Dr. med. M.-Christine Peter-Gattlen, Mitglied des Zentralvorstandes der FMH 